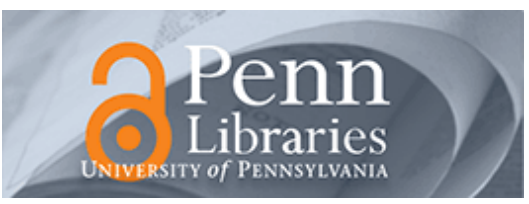

University of Pennsylvania

ScholarlyCommons

$12-2011$

\title{
On Consensus in a Correlated Model of Network Formation Based on a Polya Urn Process
}

\author{
Arastoo Fazeli \\ University of Pennsylvania \\ Ali Jadbabaie \\ University of Pennsylvania, jadbabai@seas.upenn.edu
}

Follow this and additional works at: https://repository.upenn.edu/ese_papers

Part of the Algebra Commons, Controls and Control Theory Commons, Dynamic Systems Commons, Other Applied Mathematics Commons, and the Probability Commons

\section{Recommended Citation}

Arastoo Fazeli and Ali Jadbabaie, "On Consensus in a Correlated Model of Network Formation Based on a Polya Urn Process", . December 2011.

Fazeli, A. \& Jadbabaie, A. (2011). On consensus in a correlated model of network formation based on a Polya urn process. Proceedings of the 50th IEEE Conference on Decision and Control and European Control Conference (CDC-ECC), pp.2341-2346. doi: 10.1109/CDC.2011.6161392

(C) 2011 IEEE. Personal use of this material is permitted. Permission from IEEE must be obtained for all other uses, in any current or future media, including reprinting/republishing this material for advertising or promotional purposes, creating new collective works, for resale or redistribution to servers or lists, or reuse of any copyrighted component of this work in other works.

This paper is posted at ScholarlyCommons. https://repository.upenn.edu/ese_papers/614

For more information, please contact repository@pobox.upenn.edu. 


\title{
On Consensus in a Correlated Model of Network Formation Based on a Polya Urn Process
}

\begin{abstract}
In this paper, we consider a consensus seeking process based on local averaging of opinions in a dynamic model of social network formation. At each time step, individual agents randomly choose another agent to interact with. The interaction is one-sided and results in the agent averaging her opinion with that of her randomly chosen neighbor. Once an agent chooses a neighbor, the probabilities of interactions are updated in such a way that prior interactions are reinforced and future interactions become more likely, resulting in a random consensus process in which networks are highly correlated with each other. Using results of Skyrm and Pemantle and utilizing the de Finetti representation theorem as well as properties of Polya urn processes, we show that this highly correlated process is equivalent to a mixture of i.i.d. processes whose parameters are drawn from a random limit distribution. Therefore, prior results on consensus on i.i.d. processes can be used to show consensus and to compute the statistics of the consensus value in terms of the initial conditions. We provide simple expressions for the mean and the variance of the asymptotic random consensus value in terms of the number of nodes. We also show that the variance converges to a factor of the empirical variance of the initial values that depends only on the size of the network and goes to zero as the size of the network grows.
\end{abstract}

\section{Keywords}

Consensus, Stochastic Process, Social Networks, Random Networks, Graph Theory

\section{Disciplines}

Algebra | Controls and Control Theory | Dynamic Systems | Other Applied Mathematics | Probability

\section{Comments}

Fazeli, A. \& Jadbabaie, A. (2011). On consensus in a correlated model of network formation based on a Polya urn process. Proceedings of the 50th IEEE Conference on Decision and Control and European Control Conference (CDC-ECC), pp.2341-2346. doi: 10.1109/CDC.2011.6161392

(C) 2011 IEEE. Personal use of this material is permitted. Permission from IEEE must be obtained for all other uses, in any current or future media, including reprinting/republishing this material for advertising or promotional purposes, creating new collective works, for resale or redistribution to servers or lists, or reuse of any copyrighted component of this work in other works. 


\title{
On Consensus in a Correlated Model of Network Formation Based on a Polya Urn Process
}

\author{
Arastoo Fazeli and Ali Jadbabaie
}

\begin{abstract}
In this paper, we consider a consensus seeking process based on local averaging of opinions in a dynamic model of social network formation. At each time step, individual agents randomly choose another agent to interact with. The interaction is one-sided and results in the agent averaging her opinion with that of her randomly chosen neighbor. Once an agent chooses a neighbor, the probabilities of interactions are updated in such a way that prior interactions are reinforced and future interactions become more likely, resulting in a random consensus process in which networks are highly correlated with each other. Using results of Skyrm and Pemantle and utilizing the de Finetti representation theorem as well as properties of Polya urn processes, we show that this highly correlated process is equivalent to a mixture of i.i.d. processes whose parameters are drawn from a random limit distribution. Therefore, prior results on consensus on i.i.d. processes can be used to show consensus and to compute the statistics of the consensus value in terms of the initial conditions. We provide simple expressions for the mean and the variance of the asymptotic random consensus value in terms of the number of nodes. We also show that the variance converges to a factor of the empirical variance of the initial values that depends only on the size of the network and goes to zero as the size of the network grows.
\end{abstract}

\section{INTRODUCTION}

Consensus algorithms based on local averaging have attracted a significant amount of attention in a diverse set of applications and contexts. These applications range from parallel and distributed computation [1], distributed control and coordination [2]-[4], and robotics [5] to opinion dynamics and belief formation in social networks [6], [7]. Recently, however, there has been a growing interest in studying consensus algorithms in a probabilistic setting [8]-[14], where network changes can be independent, identically distributed over time [11], ergodic-stationary [15], or Markovian [16]. In [11] the authors show that consensus is reached almost surely if and only if the network contains a directed spanning tree in expectation. The consensus value of course, will be random. In [17] the authors studied asymptotic statistics of the consensus value for a special case when the network change is governed by a randomly switching Erdos-Renyi graph process.

In many social and engineered networks, however, the interactions at any moment depend on interactions in the past, and the network change is endogenous [18], [19]. Among the studied random models of network formation, preferential attachment and strategic network formation models [20]-[23] allow for correlation in time. However, most of these models are generated according to exogenous processes

Department of Electrical and Systems Engineering and GRASP Laboratory at University of Pennsylvania. arastood seas . upenn. edu and jadbabai@seas. upenn. edu. This research was supported by ONR MURI HUNT, ONR MURI N000140810747, ARO MURI SWARMS, and AFOSR Complex Networks Program. where agents do not take part in the evolution of the social network. Recently, a simple but elegant model of social network formation was proposed by Skyrm and Pemantle in [24]. In this dynamic model of social network formation, the social network structure emerges as a consequence of the dynamic interactions of the agents. The premise of the model is that people in a new social network usually start out with no predetermined preferences for others. However, as time progresses, prior interactions reinforce relationships. The Skyrm-Pemantle's model is in some ways similar to randomized gossip algorithms [25] in which a node communicates with a randomly chosen neighbor. The key difference is that the model proposed in [24] is asymmetric, while in randomized gossip symmetric communication links are assumed. Other results on gossip algorithms include those of [26] in which the authors study three different randomized consensus algorithms; the symmetric gossip model, the ingossip model and the broadcasting model. The first model has symmetric communication links, while the other two models are asymmetric networks. It is shown that while the first model preserves the global average, the other two do not. A mean square analysis for the various algorithms is proposed as well. The in-gossip model is similar to the Skyrm-Pemantle's model with the difference that the SkyrmPemantle's model is generated by an endogenous process compared to an i.i.d. exogenous process for the in-gossip model.

In this paper, we consider consensus algorithms over a dynamic social network inspired by the Skyrm-Pemantle's model [24]. We begin with a social network with $n$ individuals and no edges. Individuals in the society select whom to visit at random and then modify their choices depending on how their choice is reinforced. Intuitively this means, people who have been visited are more likely to be visited in the future. This model seems to be a realistic model since studies have shown that in the absence of other environmental attributes, familiarity results in positive change of people's attitudes [27]. We denote this model as the "friendship model", since visiting people randomly and reinforcing those who have been visited can be thought of as making new friends in a society and developing stronger friendship with people who have been visited more often.

While considering network structural dynamics, we assume each individual in the society has a belief about some state in the world. While visiting other people, each person updates her belief according to the average of her belief and the belief of her new friend she just visited. With this simple belief dynamics, we will study whether people's beliefs will converge to the same value and if so, what the characteristic of this value is. Though at each time step the network seems 
to depend on the previous steps, we show that it can be modeled as a mixture of i.i.d. random networks; hence, we can exploit the results of consensus over i.i.d. networks. We show that agents reach consensus over this dynamic model of social network formation. Due to the potential asymmetry in pairwise communications between different agents, the asymptotic value of consensus is not guaranteed to be the average of the initial conditions. Instead, agents will asymptotically agree on a random value in the convex hull of the initial conditions. We derive closed-form expressions for the mean and the variance of the asymptotic consensus value when the underlying network evolves according to this random process.

The paper is organized as follows: In section II, we consider a dynamic model of social network formation. The interaction between any pair of agents always produces a constant reward, and so this model can be thought of as a model of uniform reinforcement. The network structural dynamics are endogenous in the sense that the interaction between any pair of agents determines the network structure. In section III, we define consensus and prove that consensus is reached in this model almost surely. In section IV, we derive an explicit expression for the mean and the variance of the limiting consensus value, and show that as the network size grows, the consensus value is concentrated around the average of the initial conditions. Section V contains simulations of our results and section VI concludes the paper.

\section{THE MODEL}

In this section we discuss a dynamic model of social network formation. The idea behind this model is that in social networks, links formed at each time step are more likely to be formed in the future. Intuitively this means that social networks formed at each time depend on social interactions in the past and will influence the future formation of the network.

We consider directed graphs $G_{t}=\left(V, E_{t}\right)$ with a fixed set of vertices $V=\{1, \ldots, n\}$ and directed edges which are formed at each time according to random dynamics. In order to model the dynamics of link formation, we consider a sequence of likelihood matrices $\{W(t)\}_{t=0}^{\infty}$ defined as follows: Let $w_{i}(t)=\left(w_{i 1}(t), \ldots, w_{i n}(t)\right)$ be the likelihood weight vector that agent $i$ assigns to other agents $j \neq i$ at time $t\left(w_{i i}(t)=0\right)$. The weight $w_{i j}(t)$ can be thought of as each agent's preference for visiting other agents in the network. Initially all likelihood weights are the same and are equal to one, i.e. $W(0)=\mathbf{1}_{n} \mathbf{1}_{n}^{T}-I_{n}$, meaning that all agents visit other agents with equal probability. In other words, people in a new social network do not have predetermined preferences.

At each time step, every agent visits another agent with a probability proportional to her assigned likelihood weight, and then forms a link with the agent she visited. We assume each agent can form only one link at each time step. Therefore, the probability of agent $i$ forming a link with agent $j \neq i$ is

$$
\operatorname{prob}(\text { agent } i \text { visits } j \text { at time } t)=p_{i j}(t)=\frac{w_{i j}(t)}{\sum_{k} w_{i k}(t)} .
$$

We assume the visit made by each agent is independent of visits made by any other agent (rows of $P(t)$ are independent). Define $A(t)=\left[a_{i j}(t)\right]$ as the adjacency matrix for the graph $G_{t}=\left(V, E_{t}\right)$ at time $t$ which is a random matrix with zero diagonal elements $\left(a_{i i}(t)=0\right)$. For off-diagonal elements we have $a_{i j}(t)=1$ if agent $i$ forms a link with agent $j$ at time step $t$ (which has the probability defined in (1)), and $a_{i j}(t)=0$ otherwise. Since at each time step each agent can visit only one agent and forms a link with her, rows of $A(t)$ consist of only one element with the value of one and the other elements are zero. Therefore, they have a categorical distribution (see Appendix for definition) with a parameter of the vector $p_{i}(t)$.

After link formations at each round, each agent increases the likelihood weight associated with the agent she visited by one. This can be thought of as the payoff of the game played by these two agents. In other words, agents who have been visited are reinforced and are more likely to be visited in the future. Therefore, the update of the likelihood matrix $W(t)$ is

$$
W(t+1)=W(t)+A(t) .
$$

Afterwards, probabilities of visits for the next round, i.e. $P(t+1)$, are modified according to the new $W(t+1)$ :

$$
p_{i j}(t+1)=\frac{w_{i j}(t+1)}{\sum_{k} w_{i k}(t+1)} .
$$

\section{CONSENSUS DYNAMICS}

We now study the consensus problem on the dynamic model of social network formation discussed in section II. Define a sequence of stochastic matrices $\{Z(t)\}_{t=0}^{\infty}$ corresponding to a sequence of random realizations of $\{A(t)\}_{t=0}^{\infty}$. For the realization of the directed graph at time $t$, we define

$$
Z(t)=\frac{I_{n}+A(t)}{2},
$$

where $A(t)$ is the adjacency matrix of the graph realization. $Z(t)$ is divided by a factor of two to ensure that it is a stochastic matrix. Adding the identity matrix to the adjacency matrix in (3) is equivalent to introducing self-loops over vertices. Consider the discrete-time linear dynamical system

$$
x(t+1)=Z(t) x(t)
$$

where $t \in\{0,1,2, \ldots\}$ is the discrete time index, $x_{i}(t) \in \mathbb{R}$ is the belief of agent $i$ at time $t$, and $\{Z(t)\}_{t=0}^{\infty}$ was defined in (3). At each time, each agent takes an average of her belief and the belief of the agent she visited at random. This probability of visits comes from (1). If agent $i$ visits agent $j$, we have

$$
x_{i}(t+1)=\frac{1}{2}\left(x_{i}(t)+x_{j}(t)\right){ }^{1}
$$

Definition 1: Dynamical system (4) reaches consensus almost surely, if for any initial state value $x(0)$

$$
\left|x_{i}(t)-x_{j}(t)\right| \rightarrow 0 \quad \mathbb{P} \text {-almost surely }
$$

as $t \rightarrow \infty$ for all $i, j \in\{1, \ldots, n\}$.

\footnotetext{
${ }^{1}$ Weighted average can be used as well.
} 
The following theorem shows almost sure consensus for the model.

Theorem 1: Dynamical system (4) with the dynamics defined in (2) reaches consensus almost surely.

Proof: The update of the weights in (2) is the same as a Polya urn process. The weight vector for each agent can be modeled as an urn with $n-1$ colors of balls where each agent is assigned to a color. There is one ball of each color in each urn at first. Then at each visit, each agent picks a ball at random, corresponding to the agent she visits, and then adds a ball of the same color to its urn. Urns belonging to different agents are independent. In [28] it is shown that for this urn process the random sequence of visits for each agent $\left(A_{i}=\left\{A_{i}(t)\right\}_{t=0}^{\infty}\right)$ is exchangeable, i.e. permuting the sequence does not change its joint probability distribution. In other words, for any finite set $\left\{k_{1}, \ldots, k_{j}\right\}$ of distinct indices, $\left(A_{i}\left(k_{1}\right), \ldots, A_{i}\left(k_{j}\right)\right)$ and $\left(A_{i}(1), \ldots, A_{i}(j)\right)$ both have the same joint probability distribution.

The de Finetti representation theorem states that the probability distribution of any infinite exchangeable sequence (not necessarily i.i.d.) is a "mixture" of probability distributions of i.i.d. sequences. Mixture here means a process whose generating parameter is random. It can be thought of as if the sequence was actually generated by first drawing a random $p$ from some probability distribution, which is the limit distribution of the original non i.i.d. sequence, and then sampling an i.i.d. sequence with the probability of $p$. Formally, the de Finetti representation theorem states that if a sequence of $A=\{A(t)\}_{t=0}^{\infty}$ is exchangeable, then there exists a distribution function $F(p)$ such that for any realization of the process

$$
\begin{aligned}
& \mathbb{P}(A(0)=a(0), A(1)=a(1), \ldots)= \\
& \int \mathbb{Q}_{i i d}(A(0)=a(0), A(1)=a(1), \ldots \mid p) d F(p),
\end{aligned}
$$

where $\mathbb{P}$ is the joint probability distribution for the sequence $A=\{A(t)\}_{t=0}^{\infty}$ and $\mathbb{Q}_{i i d}$ is the joint probability distribution with the parameter $p$ generating i.i.d. samples. For instance, if $A$ is a sequence of Bernoulli random variables (see Appendix for definition), then there exists a distribution function $F(p)$ on interval $[0,1]$ such that for any $n \geq 1$

$\mathbb{P}(A(0)=a(0), \ldots, A(n-1)=a(n-1))=\int_{0}^{1} p^{S_{n}}(1-p)^{n-S_{n}} d F$, where $S_{n}=\sum_{i=0}^{n-1} a(i)$. The distribution function $F$ is a function of the limiting frequency:

$$
\begin{aligned}
& Y=\bar{A}(\infty)=\lim _{n \rightarrow \infty} \frac{1}{n} \sum_{t=0}^{n-1} A(t), \\
& \mathbb{P}(Y \leq p)=F(p),
\end{aligned}
$$

and conditioning on $Y=p$ results in i.i.d. draws:

$$
\begin{aligned}
& \mathbb{P}(A(0)=a(0), A(1)=a(1), \ldots \mid p)= \\
& \mathbb{Q}_{i i d}(A(0)=a(0), A(1)=a(1), \ldots \mid p) .
\end{aligned}
$$

Since the sequence of visits for each agent in the model has a categorical distribution, the random sequence of visits is a mixture of i.i.d. sequences of categorical processes.
In [28] the limit distribution (mixing measure) of the urn process has been shown to be a Dirichlet distribution (see Appendix for definition). In other words, if we consider the limit distribution of the random sequence of visits, i.e. the normalized sum of random matrices $A(t)$ :

$$
\bar{A}(\infty)=\lim _{n \rightarrow \infty} \frac{1}{n} \sum_{t=0}^{n-1} A(t)
$$

then each row of the random limit matrix $\bar{A}(\infty)$ has a Dirichlet distribution with a parameter $\mathbf{1}_{n}$.

To summarize, the random sequence of visits is equivalent to a mixture of i.i.d. categorical processes with the parameter vector $\vec{p}$ drawn from the limit Dirichlet distribution. The process $Z(t)=\frac{I_{n}+A(t)}{2}$ is also a mixture of i.i.d. processes. This is true since adding a deterministic identity matrix to $A(t)$ is equivalent to having another urn with one ball in it from which never a ball is picked. Therefore, it doesn't have any effect on our probabilistic analysis. Also note that $\bar{Z}(\infty)=\frac{I_{n}+\bar{A}(\infty)}{2}$, thus, the off diagonal elements in each row of the random limit matrix $\bar{Z}(\infty)$ have a Dirichlet distribution as well.

In [11] it has been shown that for i.i.d. random processes, if the graph of the network is connected in expectation and has positive diagonals (self-loops), then dynamical system (4) reaches consensus on almost all paths. To show that we reach consensus for any i.i.d. process generated by the random vector $\vec{p}$ in the model, we take the expectation of the i.i.d. process conditioned on $\vec{p}$ as its parameter. For the diagonal elements of $\mathbb{E}(Z(t))$ we have $\mathbb{E}\left(z_{i i}(t)\right)=\frac{1}{2}$ for all $i \in\{1, \ldots, n\}$ and $t$ because of the self loop at each vertex and the normalizing factor. For all off-diagonal elements of $\mathbb{E}(Z(t))$ we have

$$
\mathbb{E}\left(z_{i j}(t) \mid \vec{p}\right)=\frac{1}{2} p_{i j}(t),
$$

where we used the fact that the expectation of the $i j$-th element of a categorical distribution with the parameter $\vec{p}$ is $p_{i j}$. Therefore, for any i.i.d. process generated by the random vector $\vec{p}$, the graph of $\mathbb{E}(Z(t))$ is a complete graph and as a result connected. Hence, the i.i.d. process reaches consensus. Now, for a sample path $Z=\{Z(t)\}_{t=0}^{\infty}$, define an event $\mathrm{A}$ as:

$$
A=\left\{Z: \prod_{t=0}^{\infty} Z(t)=\mathbf{1}_{n} d^{T} \quad \text { for some } \mathrm{d}\right\} .
$$

Since equation (5) holds for all sample paths, it also holds for an infinite length sample path whose product is a rank one matrix. Therefore, we have

$\mathbb{P}\left(Z: \prod_{t=0}^{\infty} Z(t)=\mathbf{1}_{n} d^{T}\right)=\int \mathbb{Q}_{i i d}\left(Z: \prod_{t=0}^{\infty} Z(t)=\mathbf{1}_{n} d^{T} \mid p\right) d F(p)$.

However, we know we reach consensus for any i.i.d. process, so $\mathbb{Q}_{i i d}\left(Z: \prod_{t=0}^{\infty} Z(t)=\mathbf{1}_{n} d^{T} \mid p\right)=1$. Therefore, $\mathbb{P}(A)=$ 1 and the dynamical system (4) reaches consensus with probability one.

The above argument demonstrates that consensus is reached with probability one, even though the network change is highly correlated. This is in fact due to the initial 
possibility of all interactions among agents that results in the spread of all people's ideas in the social network. The interesting question of course is, what the initial opinions tells us about the consensus value and whether the information is correctly aggregated.

\section{Statistical Analysis of the Consensus Value}

In this section we derive an explicit expression for the mean and the variance of the consensus value for the dynamic model we described in section II. The authors in [11] have found closed form formulas for the mean and the variance of the consensus value in terms of the first two moments of the i.i.d. weight matrix. The mean of the random consensus value is given by

$$
\mathbb{E}\left(x^{*}\right)=x(0)^{T} v_{1}(\mathbb{E}(Z(t))),
$$

and its variance is given by

$$
\begin{aligned}
& \operatorname{var}\left(x^{*}\right)=[x(0) \otimes x(0)]^{T} \operatorname{vec}(\operatorname{cov}(d))= \\
& {[x(0) \otimes x(0)]^{T} v_{1}(\mathbb{E}(Z(t) \otimes Z(t)))-\left[x(0)^{T} v_{1}(\mathbb{E}(Z(t)))\right]^{2},}
\end{aligned}
$$

where $v_{1}($.$) denotes the normalized left eigenvector corre-$ sponding to the unit eigenvalue, and $\otimes$ denotes the Kronecker product.

We use these two formulas to derive an explicit expression for the mean and the variance of the limiting consensus value for our model. For this purpose, we need to compute $v_{1}^{T}(\mathbb{E}(Z(t)))$ and $v_{1}^{T}(\mathbb{E}(Z(t) \otimes Z(t)))$ in (6) and (7). In order to compute $v_{1}^{T}(\mathbb{E}(Z(t)))$, we first need to compute the expectation of each entry of $Z(t)$.

The parameter $\vec{p}$ generating the process $Z(t)$ is a random vector, and conditioning on $\vec{p}$ this process is equivalent to an i.i.d. process. Therefore, in order to compute the expectation of $Z(t)$, we should use conditional expectation. For the diagonal elements of $\mathbb{E}(Z(t))$ we have $\mathbb{E}\left(z_{i i}(t)\right)=\frac{1}{2}$ for all $i \in\{1, \ldots, n\}$ and $t$. Off-diagonal elements of $\mathbb{E}(\stackrel{2}{Z}(t))$ in each row have a conditional categorical distribution; hence we have

$$
\begin{aligned}
& \mathbb{E}\left(z_{i j}(t)\right)=\mathbb{E}_{d}\left(\mathbb{E}_{c}\left(z_{i j}(t) \mid \bar{Z}(\infty)\right)\right)=\frac{1}{2} \mathbb{E}_{d}\left(\bar{a}_{i j}(\infty)\right) \\
& =\frac{\alpha_{j}}{2\left(\sum_{i=1}^{n} \alpha_{i}\right)}=\frac{1}{2(n-1)}
\end{aligned}
$$

where the inner expectation, i.e. $\mathbb{E}_{c}$, is with respect to the categorical distribution and the outer expectation, i.e. $\mathbb{E}_{d}$, is with respect to the limit Dirichlet distribution with the parameter vector $\alpha=\left(\alpha_{1}, \alpha_{2}, \ldots, \alpha_{n}\right)=\mathbf{1}_{n}$. Using this fact, it is straightforward to see that the vector $\mathbf{1}_{n}$ satisfies the eigenvalue equation $\mathbf{1}_{n}^{T} \mathbb{E}(Z(t))=\mathbf{1}_{n}^{T}$, thus,

$$
v_{1}(\mathbb{E}(Z(t)))=\frac{1}{n} \mathbf{1}_{n} .
$$

Therefore, as expected, the mean of the random consensus value is equal to the average of $x(0)$, i.e. $\mathbb{E}\left(x^{*}\right)=$ $\frac{1}{n} \sum_{i=1}^{n} x_{i}(0) \triangleq \bar{x}(0)$. The other term in the expression of the variance that we need to compute is $v_{1}(\mathbb{E}(Z(t) \otimes Z(t)))$. In order to compute this vector, we first compute the entries of the matrix $\mathbb{E}(Z(t) \otimes Z(t))$. For this purpose, we divide the matrix $Z(t) \otimes Z(t)$ into $n \times n$ blocks where each of them has $n \times n$ entries. Define block $B(t)^{i j}$ as follows:

$$
\begin{aligned}
& B(t)^{i j} \triangleq\left[(Z(t) \otimes Z(t))_{k l}\right], \\
& k \in\{n(i-1)+1, \ldots, n i\}, \\
& l \in\{n(j-1)+1, \ldots, n j\},
\end{aligned}
$$

where $(Z(t) \otimes Z(t))_{k l}$ is the $k l$-th element of $Z(t) \otimes Z(t)$. Now, we compute $\mathbb{E}(Z(t) \otimes Z(t))_{k l}$ for an arbitrary block $B(t)^{i j}$. From the definition of the Kronecker product we have $B(t)^{i j}=z_{i j}(t) Z(t)$, where $z_{i j}(t)$ is the $i j$-th element of $Z(t)$. For the $k l$-th entry of blocks $B(t)^{i i}$ we have $z_{i i}(t)=\frac{1}{2}$; therefore, $\mathbb{E}\left(B_{k l}(t)^{i i}\right)=\frac{1}{4(n-1)}$. For the $j j$-th entry of blocks $B(t)^{i i}$ we have $z_{i i}(t)=\frac{1}{2}$ and $z_{j j}(t)=\frac{1}{2}$, so obviously $\mathbb{E}\left(B_{j j}(t)^{i i}\right)=\frac{1}{4}$. Note that the rows of $Z(t)$ are independent; therefore, for all off-diagonal entries of all rows of $B(t)^{i j}$, except the $i$-th row, we have

$$
\mathbb{E}\left(B_{k l}(t)^{i j}\right)=\mathbb{E}\left(z_{i j}(t)\right) \mathbb{E}\left(z_{k l}(t)\right)=\frac{1}{4(n-1)^{2}} .
$$

For all off-diagonal entries of the $i$-th row of $B(t)^{i j}$ we should exploit the concept of variance and covariance since there is an inherent dependence. For the $i k$-th entry of $B(t)^{i j}$ we have

$$
\begin{aligned}
& \mathbb{E}\left(B_{i k}(t)^{i j}\right)=\mathbb{E}_{d}\left[\mathbb{E}_{c}\left(z_{i j}(t) z_{i k}(t) \mid \bar{Z}(\infty)\right)\right]= \\
& \mathbb{E}_{d}\left[\operatorname{cov}\left(\bar{z}_{i j}(t), \bar{z}_{i k}(t)\right)+\mathbb{E}_{d}\left(\bar{z}_{i j}(t)\right) \mathbb{E}_{d}\left(\bar{z}_{i k}(t)\right)\right]= \\
& \frac{1}{4} \mathbb{E}_{d}\left[-\bar{a}_{i j}(\infty) \bar{a}_{i k}(\infty)+\bar{a}_{i j}(\infty) \bar{a}_{i k}(\infty)\right]=0 .
\end{aligned}
$$

For the $i j$-th entry of $B(t)^{i j}$ we have

$$
\begin{aligned}
& \mathbb{E}\left(B_{i j}(t)^{i j}\right)=\mathbb{E}_{d}\left[\mathbb{E}_{c}\left(z_{i j}(t)^{2} \mid \bar{Z}(\infty)\right)\right]=\mathbb{E}_{d}\left[\operatorname { v a r } \left(\bar{z}_{i j}(t)+\right.\right. \\
& \left.\left(\mathbb{E}_{d}\left(\bar{z}_{i j}(t)\right)\right)^{2}\right]=\frac{1}{4} \mathbb{E}_{d}\left[\bar{a}_{i j}(\infty)\left(1-\bar{a}_{i j}(\infty)\right)+\bar{a}_{i j}(\infty)^{2}\right]= \\
& \frac{1}{4} \mathbb{E}_{d}\left(\bar{a}_{i j}(\infty)\right)=\frac{1}{4(n-1)} .
\end{aligned}
$$

Finally, for all diagonal entries $k k$ of $B(t)^{i j}$ we have $z_{k k}(t)=\frac{1}{2}$; therefore, $\mathbb{E}\left(B_{k k}(t)^{i j}\right)=\frac{1}{4(n-1)}$.

We now exploit the identified pattern to explicitly compute the normalized left eigenvector $v_{1}(\mathbb{E}(Z(t) \otimes Z(t)))$ using the same approach that is used in [29].

Lemma 1: The normalized left eigenvector of $\mathbb{E}(Z(t) \otimes$ $Z(t))$ corresponding to its unit eigenvalue is given by

$v_{1}(\mathbb{E}(Z(t) \otimes Z(t)))=\frac{1}{n \delta}\left[\rho\left(\mathbf{1}_{n} \otimes \mathbf{1}_{n}\right)+(1-\rho) \sum_{i=1}^{n}\left(e_{i} \otimes e_{i}\right)\right]$,

where $\rho$ and $\delta$ depend on $n$ as follows:

$$
\rho \triangleq \frac{2 n-2}{3 n-4}, \quad \delta \triangleq 1+(n-1) \rho
$$

Proof: First, notice that $\mathbb{E}(Z(t) \otimes Z(t))$ is a stochastic matrix. Therefore, it has a unique left eigenvector corresponding to its unit eigenvalue, which means that $v_{1}(\mathbb{E}(Z(t) \otimes Z(t)))$ is well defined. We now show that the pattern of this left eigenvector is of the form

$v_{1}(\mathbb{E}(Z(t) \otimes Z(t)))=\frac{1}{\delta}\left[\alpha\left(\mathbf{1}_{n} \otimes \mathbf{1}_{n}\right)+(\beta-\alpha) \sum_{i=1}^{n}\left(e_{i} \otimes e_{i}\right)\right]$, 
for some positive numbers $\alpha$ and $\beta$. Notice that all entries of this vector are equal to $\alpha$, except for the ones indexed $1+r(n+1)$ for $r \in\{0, \ldots, n-1\}$, which are equal to $\beta$. To show that the eigenvector we are looking for is indeed of the given pattern, we pre-multiply $\mathbb{E}(Z(t) \otimes Z(t))$ by $v_{1}(\mathbb{E}(Z(t) \otimes Z(t)))$. We notice that we have two types of linear equations: The first type is a set of $n$ linear equations for the $k$-th column of the matrix $\mathbb{E}(Z(t) \otimes Z(t))$ where $k=1+r(n+1)$ and $r \in\{0, \ldots, n-1\}$. In this case, we have

$$
v_{1}(\mathbb{E}(Z(t) \otimes Z(t)))^{T}(\mathbb{E}(Z(t) \otimes Z(t)))_{k}=\beta,
$$

the second type is a set of $n^{2}-n$ linear equations for all other $\left(n^{2}-n\right)$ columns of $\mathbb{E}(Z(t) \otimes Z(t))$, where

$$
v_{1}(\mathbb{E}(Z(t) \otimes Z(t)))^{T}(\mathbb{E}(Z(t) \otimes Z(t)))_{k}=\alpha .
$$

Exploiting the identified pattern for $\mathbb{E}(Z(t) \otimes Z(t))$ and solving equations (11) and (12), we get the closed form solution $\alpha=2 n-2$ and $\beta=3 n-4$. Hence,

$v_{1}(\mathbb{E}(Z(t) \otimes Z(t)))=\frac{1}{n \delta}\left[\rho\left(\mathbf{1}_{n} \otimes \mathbf{1}_{n}\right)+(1-\rho) \sum_{i=1}^{n}\left(e_{i} \otimes e_{i}\right)\right]$,

where $\rho=\frac{\alpha}{\beta}=\frac{2 n-2}{3 n-4}$ and $\delta(n)$ defined in (10) is a normalizing factor guaranteeing that the elements of the vector $v_{1}$ sum up to one.

Now that we have derived explicit expressions for the eigenvectors (8) and (9), we can compute a closed-form expression for the variance of the limiting consensus value in terms of $n$.

Theorem 2: The variance of the asymptotic consensus value $x^{*}$ of the dynamical system defined in (4) is given by

$$
\operatorname{var}\left(x^{*}\right)=\frac{1-\rho}{n \delta} \sum_{i=1}^{n}\left[x_{i}(0)-\bar{x}(0)\right]^{2},
$$

where $\rho$ and $\delta$ are defined in (10).

Proof: First, from (8), we have

$$
\left[x(0)^{T} v_{1}(\mathbb{E}(Z(t)))\right]^{2}=\left(\frac{1}{n} \sum_{i=1}^{n} x_{i}(0)\right)^{2}=(\bar{x}(0))^{2} .
$$

On the other hand, from (9), we have

$$
\begin{aligned}
& {[x(0) \otimes x(0)]^{T} v_{1}(\mathbb{E}(Z(t) \otimes Z(t)))=} \\
& \frac{1}{n \delta}[x(0) \otimes x(0)]^{T}\left[\rho\left(\mathbf{1}_{n} \otimes \mathbf{1}_{n}\right)+(1-\rho) \sum_{i=1}^{n}\left(e_{i} \otimes e_{i}\right)\right]= \\
& \frac{\rho}{n \delta}\left[x(0)^{T} \mathbf{1}_{n}\right] \otimes\left[x(0)^{T} \mathbf{1}_{n}\right]+\frac{1-\rho}{n \delta} \sum_{i=1}^{n}\left[x(0)^{T} e_{i}\right] \otimes\left[x(0)^{T} e_{i}\right],
\end{aligned}
$$

where we have used the fact that $(A \otimes B)(C \otimes D)=A C \otimes$ $B D$. Since the Kronecker terms in the last expression are scalars, we have

$[x(0) \otimes x(0)]^{T} v_{1}(\mathbb{E}(Z(t) \otimes Z(t)))=\frac{\rho}{\delta} n \bar{x}(0)^{2}+\frac{1-\rho}{n \delta} \sum_{i=1}^{n} x_{i}(0)^{2}$,

and therefore from (7)

$$
\operatorname{var}\left(x^{*}\right)=\left(\frac{\rho}{\delta} n-1\right) \bar{x}(0)^{2}+\frac{1-\rho}{n \delta} \sum_{i=1}^{n} x_{i}(0)^{2} .
$$

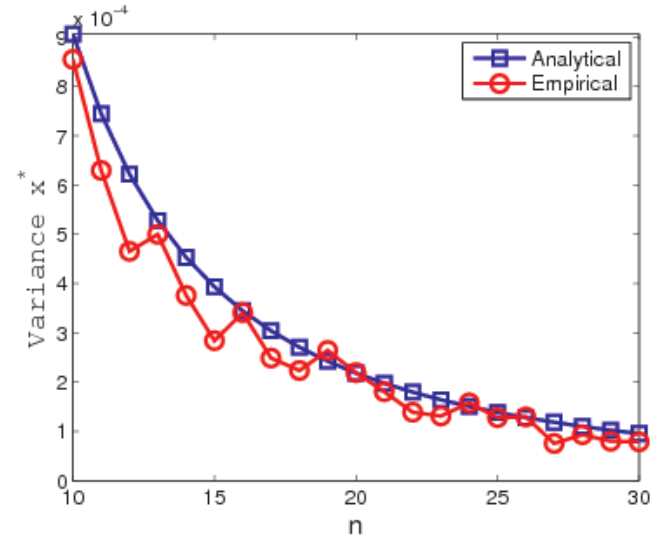

Fig. 1. Comparison between the empirical variance and the analytical variance for $n$ in the interval [10:30]

By adding and subtracting $\left(\frac{1-\rho}{\delta}\right) \bar{x}(0)^{2}$, the expression for the variance can be rewritten as

$\operatorname{var}\left(x^{*}\right)=\left(\frac{(n-1) \rho+1-\delta}{\delta}\right) \bar{x}(0)^{2}+\frac{1-\rho}{n \delta} \sum_{i=1}^{n}\left(x_{i}(0)-\bar{x}(0)\right)^{2}$.

Since $\delta=1+(n-1) \rho$, as defined in (10), the first term on the right-hand-side of the above expression is equal to zero. This proves the theorem.

Remark 1: Equation (13) shows that, given the size of the random graph $n$, the variance of the limiting consensus value $x^{*}$ is equal to the empirical variance of the initial conditions multiplied by the factor $\frac{1-\rho}{\delta}$, which only depends on the size of the network. More importantly, as the network becomes larger, the variance of the consensus value goes to zero, demonstrating that (at least for linear models), the information is correctly aggregated and naive learning in the sense of Golub and Jackson [7] occurs, even though the update is rather simple and myopic and the neighbors' opinions are not aggregated in a rational way.

\section{Numerical Simulations}

We now present a simulation that illustrates the decay in variance as a function of the size of the network. In Fig.1, we compare the analytical expression for the variance in (13) with the empirical variance obtained from 100 realizations of the random consensus algorithm for $n$ in a specific range of network sizes. In this figure, we plot both the analytical and empirical variances when the network size $n$ goes from 10 to 30 nodes. The initial conditions for each network size are given by $x_{i}(0)=\frac{i}{n}$, for $i \in\{1, \ldots, n\}$. For this specific choice of the initial states the empirical variance does not scale with $n$; therefore, the comparison of the analytical and the empirical variance is more meaningful. We can see that for larger $n$ the variance decreases with the network size $n$. It is easy to see from (13) that this variance tends to zero asymptotically as $n \rightarrow \infty$ at a rate of $\frac{1}{n}$.

\section{CONCLUSIONS}

We studied the mean and the variance of the consensus value in a gossip-like opinion aggregation process in which the network is formed randomly, and interactions taking 
place within the network determine the network structure. Individuals in the society select whom to visit at random and then modify their choices depending on how their choice is reinforced. The visit made by each agent is independent of visits made by any other agent. At each time, each agent takes an average of her belief and the belief of the agent she visited at random. Although the realizations of the network are not i.i.d., we showed that the sequence of visits for each agent is exchangeable. Therefore, the random sequence of visits is equivalent to a mixture of i.i.d. categorical processes. Using recent results on consensus for i.i.d. processes, we showed that the consensus algorithm results in asymptotic agreement almost surely. The asymptotic value of consensus was shown to be a random value in the convex hull of the initial conditions. We exploited results for the mean and the variance of the consensus value in terms of the first two moments of the i.i.d. weight matrix in order to drive closedform expressions for the expectation and the variance as functions of the number of nodes. While the expectation of the distribution of the consensus value is simply the mean of the initial conditions, the variance of the consensus value is the empirical variance of the initial conditions multiplied by a factor that depends only on $n$. This factor goes to zero as $n$ goes to infinity.

\section{APPENDIX}

In this section, we define the probability distributions used throughout the paper.

Definition 2: Bernoulli distribution:

A random variable $X$ has a Bernoulli distribution with the parameter $p$, if $X=1$ with probability $p$ and $X=0$ with probability $1-p$.

Definition 3: Categorical distribution:

A categorical distribution is a generalization of the Bernoulli distribution where a trial results in exactly one of some fixed finite number $n$ of possible outcomes, with probabilities $p_{1}, \ldots, p_{n}$ for each outcome respectively, so that $\sum_{i=1}^{n} p_{i}=1$. Let the random variables $X_{i}=1$ if outcome number $i$ is observed, and $X_{i}=0$ otherwise. The vector $X=\left(X_{1}, \ldots, X_{n}\right)$ follows a categorical distribution with parameters $p$ where $p=\left(p_{1}, \ldots, p_{n}\right)$.

Definition 4: Dirichlet distribution:

A Dirichlet distribution of order $k \geq 2$ with parameters $\alpha_{1}, \ldots, \alpha_{k}>0$ has a probability density function with respect to the Lebesgue measure on the Euclidean space $\mathbb{R}^{k-1}$ given by

$$
f\left(x_{1}, \ldots, x_{k} ; \alpha_{1}, \ldots, \alpha_{k}\right)=\frac{1}{\mathbf{B}(\alpha)} \prod_{i=1}^{k} x_{i}^{\alpha_{i}-1}
$$

for all $x_{1}, \ldots, x_{k-1}>0$ satisfying $x_{1}+\ldots+x_{k-1}<1$, where $x_{k}$ is an abbreviation for $1-x_{1}-\ldots-x_{k-1}$. The density is zero outside this open $(k-1)$-dimensional simplex. The normalizing constant is the multinomial beta function, which can be expressed in terms of the gamma function:

$$
\mathbf{B}(\alpha)=\frac{\prod_{i=1}^{k} \Gamma\left(\alpha_{i}\right)}{\Gamma\left(\sum_{i=1}^{k} \alpha_{i}\right)}, \quad \alpha=\left(\alpha_{1}, \ldots, \alpha_{k}\right) .
$$

\section{REFERENCES}

[1] J. Tsitsiklis, "Problems in decentralized decision making and computation," Ph.D. dissertation, 1984.

[2] A. Jadbabaie, J. Lin, and A. Morse, "Coordination of groups of mobile autonomous agents using nearest neighbor rules," IEEE Transactions on Automatic Control, vol. 48, no. 6, pp. 988-1001, 2003.

[3] R. Olfati-Saber and R. Murray, "Consensus problems in networks of agents with switching topology and time-delays," IEEE Transactions on Automatic Control, vol. 49, no. 9, pp. 1520-1533, 2004.

[4] L. Moreau, "Stability of multiagent systems with time-dependent communication links," IEEE Transactions on Automatic Control, vol. 50, no. 2, pp. 169-182, 2005.

[5] J. Cortes, S. Martinez, and F. Bullo, "Analysis and design tools for distributed motion coordination," in American Control Conference, 2005. Proceedings of the 2005, pp. 1680-1685, 2005.

[6] M. DeGroot, "Reaching a consensus," Journal of the American Statistical Association, vol. 69, no. 345, pp. 118-121, 1974.

[7] B. Golub and M. Jackson, "Naive Learning in Social Networks and the Wisdom of Crowds," American Economic Journal: Microeconomics, vol. 2, no. 1, pp. 112-149, 2010.

[8] Y. Hatano and M. Mesbahi, "Agreement over random networks," IEEE Transactions on Automatic Control, vol. 50, no. 11, pp. 1867-1872, 2005.

[9] C. Wu, "Synchronization and convergence of linear dynamics in random directed networks," IEEE Transactions on Automatic Control, vol. 51 , no. 7, pp. 1207-1210, 2006.

[10] M. Porfiri and D. Stilwell, "Consensus seeking over random weighted directed graphs," IEEE Transactions on Automatic Control, vol. 52, no. 9, pp. 1767-1773, 2007.

[11] A. Tahbaz-Salehi and A. Jadbabaie, "A necessary and sufficient condition for consensus over random networks," IEEE Transactions on Automatic Control, vol. 53, no. 3, pp. 791-795, 2008.

[12] G. Picci and T. Taylor, "Almost sure convergence of random gossip algorithms," in IEEE Conf. on Decision and Control, pp. 282-287, 2007.

[13] D. Acemoglu, A. Ozdaglar, and A. ParandehGheibi, "Spread of (mis) information in social networks," Games and Economic Behavior, 2010.

[14] B. Touri and A. Nedich, "On ergodicity, infinite flow and consensus in random models," Automatic Control, IEEE Transactions on, no. 99, pp. $1-1,2010$.

[15] A. Tahbaz-Salehi and A. Jadbabaie, "Consensus over ergodic stationary graph processes," IEEE Transactions on automatic Control, vol. 55 , no. 1 , pp. $225-230,2010$.

[16] M. Ion and B. John, "Convergence results for the linear consensus problem under Markovian random graphs," 2009.

[17] V. Preciado, A. Tahbaz-Salehi, and A. Jadbabaie, "Variance analysis of randomized consensus in directed switching random networks," in Proceedings of the American Control Conference, (Baltimore, MD), June 2010.

[18] R. Hegselmann and U. Krause, "Opinion dynamics and bounded confidence models, analysis, and simulation," Journal of Artifical Societies and Social Simulation (JASSS) vol, vol. 5, no. 3, 2002.

[19] V. Blondel, J. Hendrickx, and J. Tsitsiklis, "On Krause's multi-agent consensus model with state-dependent connectivity," IEEE Transactions on Automatic Control, vol. 54, no. 11, pp. 2586-2597, 2009.

[20] M. Jackson, Social and economic networks. Princeton University Press, 2008.

[21] T. Schelling, "Models of segregation," Am. Econ. Rev. Pap. Proc. 59, pp. 488-493, 1969.

[22] T. Schelling, "On the ecology of micromotives," The public interest, vol. 25, no. 1971, pp. 59-98, 1971.

[23] J. Epstein and R. Axtell, Growing artificial societies. MIT press Cambridge, MA, 1996.

[24] B. Skyrms and R. Pemantle, "A dynamic model of social network formation," Adaptive Networks, pp. 231-251, 2009.

[25] S. Boyd, A. Ghosh, B. Prabhakar, and D. Shah, "Randomized gossip algorithms," IEEE Transactions on Information Theory, vol. 52, no. 6 , pp. 2508-2530, 2006.

[26] F. Fagnani and S. Zampieri, "Randomized consensus algorithms over large scale networks," Selected Areas in Communications, IEEE Journal on, vol. 26, no. 4, pp. 634-649, 2008.

[27] R. Zajonc, "Attitudinal effects of mere exposure. 1," Pers. Soc. Psycho, vol. 9 , no. 2 pt $2,1968$.

[28] N. Johnson and S. Kotz, Urn models and their application: an approach to modern discrete probability theory. Wiley, New York, 1977.

[29] V. Preciado, A. Tahbaz-Salehi, and A. Jadbabaie, "Variance analysis of randomized consensus in switching directed networks," in American Control Conference (ACC), 2010, pp. 6330-6335, IEEE, 2010. 